\title{
El proceso de formación del profesional en la educación superior basado en competencias: el desafío de su calidad, en busca de una mayor integralidad de los egresados*
}

\author{
The process of formation of professional of higher education \\ based on competence: the challenge of the quality in search \\ of a better integration of graduates
}

Recibido: 15 de octubre de 2009 - Revisado: 2o de noviembre de 2009 - Aceptado: 16 de diciembre de 2009

René Valera Sierra**

\section{Resumen}

Se aporta una reflexión teórica acerca del Proceso de Formación del Profesional en la Educación Superior, en la aspiración de una educación integral que permita formar un profesional comprometido social y profesionalmente, flexible y trascendente, para lo que se requiere de un diseño curricular basado en competencias.

Palabras clave

Proceso de Formación del Profesional, currículo basado en competencias, modelo curricular para la formación de competencias en la Educación Superior.

\footnotetext{
Abstract

This paper provides a theoretical reflection about the Process of Professional Training in Higher Education, the aim of a comprehensive education designed to build a socially and professionally committed professional, flexible and transcendent, to what is required of a competency-based curriculum .

Keywords

Process of Professional Training, Curriculum Based on Competency Model Curriculum for the training of skills in higher education.
}

* Artículo resultado del Proyecto de Investigación: La Formación del Profesional en la Educación Superior Cubana. Un modelo curricular con bases en Competencias. CEES Manuel F. Gran, Universidad de Oriente. República de Cuba. **Ph.D. Profesor Titular. Universidad de La Habana, Cuba.

Correo electrónico:

revalera@yahoo.com.au 


\section{Introducción}

Las universidades enfrentan en la actualidad el gran reto de ampliar su capacidad de respuesta a las exigencias sociales, a las crecientes demandas que afrontan los profesionales en formación, que sean capaces de insertarse plenamente en los procesos sociales, productivos y científicos en un contexto complejo, caracterizado por las desiguales situaciones económicas, los vertiginosos cambios tecnológicos y la amplia diversidad sociocultural.

Todas estas exigencias que enfrenta la universidad como institucion de creación, preservación y promoción de la cultura llevan al debate acerca de la cuestión de la calidad de los procesos a través de los cuales se forman los profesionales, en busca de la eficiencia de sus resultados y eficacia en la consecución de los fines que la sociedad les demanda, todo lo cual implica revisar los criterios con los que se proyectan, planifican, diseñan y estructuran los planes y programas de estudio en la educación superior.

La ponencia es resultado de la tesis doctoral del autor, como parte del proceso de investigación multidisciplinaria que desde el Centro de Estudios de Educación Superior (CEES) de la Universidad de Oriente, en la República de Cuba, con el propósito de contribuir al debate actual acerca la calidad del proceso formativo que desarrollan las universidades en busca de una mayor integralidad de sus egresados, destacando las necesarias relaciones que se deben establecer entre los conceptos: profesión, formación del profesional, competencias profesionales y sus expresiones en el currículo universitario. Se ha empleado, en lo fundamental, la metodología de investigación cuantitativa y sus resultados han sido ampliamente introducidos en la práctica del proceso formativo de los profesionales en la educación superior cubana y de otros países de América Latina.

\section{La Universidad y el desafío de la calidad del proceso formativo}

Es por todos conocido que en la actualidad se están produciendo grandes transformaciones en el orden económico, político y social a nivel mundial, que tienen un impacto en la concepción del hombre y su relación con el mundo evidenciándose importantes cambios en la educación, tanto en su concepción general, como en los enfoques pedagógicos y los contenidos curriculares, tomando significación lo axiológico y actitudinal, con un énfasis en lo valorativo sin que ello implique minimizar lo cognitivo, todo ello como resultado de una visión holística del proceso formativo.

Los cambios de paradigmas de la educación, que responden a las condicionantes planteadas anteriormente, implican a su vez cambios en el significado de los conceptos y categorías, así como de la forma de relacionarlos; lo que se expresa en cambios epistemológicos, axiológicos y actitudinales frente a los fenómenos o situaciones que se interpretan e investigan desde los nuevos paradigmas y que modifican la forma de desarrollar o interpretar los procesos.

En este sentido, hay que reconocer que uno de los problemas más debatidos en este ámbito es la cuestión de la calidad de las acciones humanas para alcanzar mayor eficiencia y eficacia en sus resultados, para lo que se han acuñado diferentes categorías que intentan expresar de mejor manera esta intencionalidad, provocando la asunción de posiciones diversas en cuanto al diseño del proceso formativo en el ámbito universitario. Siendo así, es lícito expresar nuestra percepción del problema con el ánimo de contribuir de manera modesta reasignar significados a determinados conceptos que en la actualidad son utilizados en la academia, aunque no necesariamente con igual significado, teniendo impactos diversos en la teoría y práctica pedagógica en la educación superior. 
Se reconoce el proceso de formación del profesional que se desarrolla en la educación superior como un espacio de construcción de significados y sentidos entre los sujetos participantes que implica el desarrollo humano progresivo, lo que se puede explicar desde un modelo pedagógico que reconozca este proceso como un proceso consciente, complejo, holístico y dialéctico.

La formación del profesional constituye, por lo tanto, el proceso en el que los sujetos desarrollan el compromiso social y profesional, la flexibilidad ante la cultura, la trascendencia en su contexto, toda vez que elevan su capacidad para la reflexión divergente y creativa, para la evaluación crítica y autocrítica, para solucionar problemas, tomar decisiones y adaptarse flexiblemente a un mundo cambiante. Estamos asumiendo que alcanzar una integralidad en la formación profesional a nivel universitario implica, ante todo, formar un profesional comprometido con su labor y sociedad en que se inserta, flexible y trascendente, independientemente de la especificidad que impone cada profesión y sus contextos.

Es necesario que el currículo universitario contenga las cualidades que se aspira formar en los estudiantes universitarios, con lo cual se puede precisar cuál es el tipo de profesional que se quiere formar y cómo se estructura esta aspiración en todo el currículo, permitiendo cumplir su encargo de orientador de la dinámica de la formación de los profesionales sobre la base de dichas cualidades. Es importante señalar que estas no se identifican con el sistema de valores profesionales a formar, aunque lo contienen.

Coincidimos con el pedagogo Gimeno Sacristán cuando señala que "los debates esenciales en torno a los currículos en la actualidad, como no podía ser de otro modo, están muy estrechamente relacionados con los cambios culturales, políticos, sociales y económicos que están afectando a las sociedades desarrolladas $y$ que tienen como primera consecuencia la revisión del papel asignado a la escolarización y a las relaciones entre esta y los diferentes aspectos que en ella se entrecruzan: profesores, organizaciones, relaciones con la comunidad. etc."'.

Los cambios curriculares en la educación superior deben ser expresión de la contextualización de las universidades de acuerdo con la complejidad de los escenarios en los que se desarrolla. El entorno económico, político y sociocultural condiciona los procesos educativos en las universidades, sin embargo la universidad no debe asumir una posición adaptativa al entorno, sino que debe investigarlo con profundidad para generar los procesos de su transformación.

El perfeccionamiento del currículo universitario tendiente a una integralidad en la formación del profesional debe tener en cuenta "los avances científicos y tecnológicos, redefinir o rescatar los valores humanos y sociales, centrar los procesos educativos en la formación integral de las personas, hacer de los centros educativos verdaderos proyectos culturales, formar líderes para producir la transformación, partir de la realidad que ofrece el entorno y definir los cambios a la misma, investigar sobre los entornos socioculturales y definir alternativas de solución a los problemas encontrados, construir nuevos modelos pedagógicos y operarlos en los centros educativos mediante estrategias didácticas, transformar las formas tradicionales de administración, generar una cultura organizacional educativa $\mathrm{y}$, el más importante de todos, responder adecuadamente al nuevo orden político, social y económico internacional desde la gestión curricular"².

\section{La formación profesional basada en competencias}

La realidad educativa actual, en el empeño de que los procesos formativos respondan de manera más pertinente a las exigencias sociales, económicas y productivas de nuestros países, 
impone la necesidad de que los sujetos en formación demuestren con mayor efectividad el resultado de sus aprendizajes.

El proceso de formación de los profesionales, como todos los que involucran al hombre, es complejo, multidimensional y en tanto realidad objetiva no puede interpretarse desde una sola dimensión, por el contrario, se tiene que interpretar desde una perspectiva dialéctica y multidimensional, nutriéndose de la diversidad.

La formación de competencias es un proceso constructivo, socializado, que solo es posible en un espacio interdisciplinar partiendo de una concepción participativa y no directiva del proceso, con el convencimiento de que el contenido es socialmente construido e históricamente desarrollado $\mathrm{y}$, por ende, sus resultados, competencias indispensables en el profesional, se alcanzan mediante un proceso en el que se trabaja, de manera interrelacionada, los núcleos de conocimientos, las habilidades generalizadas y los valores profesionales y sociales, donde lo interdisciplinario se manifestará en lo académico, lo investigativo y lo laboral.

No existe una definición única del término 'competencia' y cada autor enfatiza determinadas cualidades de acuerdo con sus intenciones. Como bien plantea el Dr. Roberto Corral, la mayoría de las definiciones la comprenden como unidades de actuación que expresan lo que una persona debe saber y poder hacer para desarrollar y mantener un nivel de desempeño eficiente en su labor, incluyendo aspectos cognitivos, afectivos, conductuales y de experiencias (Coral, 2004).

La revisión de la mayoría de las conceptualizaciones acerca de las competencias permite adelantar que esta noción trae consigo una nueva aproximación al desarrollo humano con importantes implicaciones en el campo educativo, es la posibilidad para desarrollar en los educandos la capacidad para el análisis, la crítica y el razonamiento a través de la construcción significativa del conocimiento y de la formación para la vida ciudadana.

De lo que se trata es que las transformaciones que tengan lugar en los sistemas educativos vayan más a cambiar las concepciones y estilos acerca de cómo se organizan y ejecutan los procesos de aprendizaje que a recrear términos y conceptos. Por tanto, incorporar el concepto de competencias a la práctica educativa significa que el resultado va a estar ligado a acciones concretas, no como efecto de un aprendizaje tradicional, sino un aprendizaje donde se acrecienten las capacidades humanas mediante el desarrollo integrado de las dimensiones cognoscitivas de la personalidad, en el cual la búsqueda, la indagación, el uso de métodos científicos caracterice la solución de los problemas.

Los procesos de formación de los profesionales orientados al desarrollo de competencias emergen para hacer de la educación un servicio más pertinente a las demandas sociales, donde adquiere un significado el ser y saber cómo sobre el saber y hacer qué, capaz de ofrecer a los estudiantes aprendizajes socialmente significativos que los habiliten para operar con eficacia en el contexto específico de las dificultades y los retos propios de la época y del país.

Trabajar un currículo basado en competencias implica especificar desde qué teoría de la cognición se asume el proceso de enseñanza-aprendizaje y conceptualizar de manera coherente sobre actitudes e inteligencia. La palabra 'competencia' no se asume en este contexto como el mero entrenamiento para desarrollar habilidades y destrezas, lo que lleva a la ejecución de tareas asignadas por otro, sin protagonismo de quien las ejecuta. Desde esta perspectiva las competencias son cognoscibles y objeto de interpretación y construcción, y tienen una connotación axiológica que conlleva a una responsabilidad. 
La formación de las competencias que el individuo construye responde a estructuras complejas, tienen su individualidad que las hace diferenciables en cada sujeto, pero este, como ser social, las construye en su relación con los demás sujetos.

Así entonces, las competencias se asumen como configuraciones, construidas y desarrolladas, con un nivel de incertidumbre en sus resultados, dependiendo de los factores que inciden en el proceso, tanto en los aspectos contextuales como históricos presentes en cada proceso de construcción. Implican lo conceptual, lo metodológico, lo axiológico y lo actitudinal y no como compartimentos, separados unos de otros, porque se estaría fragmentando la unidad conceptual y metodológica de la competencia y se estaría dividiendo también la integralidad cognoscitiva del estudiante. Las competencias también pueden ser aprehendidas, nadie por competente que llegue a ser, lo fue al nacer; solo la vida en la sociedad le permite la construcción de las competencias.

Partiendo entonces de la competencia como objeto de formación de los profesionales y como un constructo negociado, se comprende como un ser, un saber y un hacer personal en un contexto histórico concreto determinado, haciendo referencia a la capacidad de tomar decisiones adecuadas en un ámbito definido. De acuerdo con el Dr. Homero Fuentes, 1998; se valora la competencia, asociada directamente al desempeño, expresado concretamente en la manifestación de los recursos con que cuenta el estudiante, el futuro profesional para realizar una tarea $\mathrm{o}$ actividad, enfrentar una situación de manera particular y crítica; se deduce entonces que la competencia presupone:

- Un grado de dominio y versatilidad conceptual (explícito o implícito) en una rama del conocimiento.

- La puesta en acción de los conocimientos, reflejada en apropiación de ideas y contenidos de esa rama.
- Un proceso de selección de alternativas de actuación $\mathrm{y}$ toma de decisiones.

- La pertinencia de su aplicabilidad en un contexto determinado.

Un proceso por competencias no propone aprendizajes fragmentarios, actitudes, destrezas y conocimientos aislados que se suman sin articularse entre sí. Todo lo contrario, integran de un modo peculiar destrezas, actitudes, conocimientos y habilidades, pero sin reducirse a estas.

En el proceso por competencias se busca enriquecer un ser, sustentado en un saber y un hacer. Por tanto, coloca a los estudiantes en situación de independencia transformadora al hacer, donde desarrollen y usen destrezas mentales y operativas pero en función de obtener un resultado. Que interpreten información pero para emplearla, y que adopten determinadas actitudes en función de resolver una situación. Que reflexionen sobre el proceso de sus propios aprendizajes y se apropien conscientemente de las capacidades desplegadas, en tanto comprueben que les sirven para mejorar su capacidad de interacción con el medio.

En otras palabras, tanto en el proceso de aprender a actuar competentemente en un campo determinado como en el mismo desempeño logrado, las actitudes y valores, el saber y el hacer interactúan de una manera contextualizada y por tanto específica, integrándose de modo cada vez superior. Lo que hace muy diferente este proceso, basado en competencias, al centrado en los objetivos, no obstante no se excluye la categoría objetivo dentro de las configuraciones del proceso.

En la formación por competencias se destacan los siguientes aspectos:

Disposición para aprender. Los estudiantes se comprometen con un proceso de aprendizaje solo si ello tiene un sentido, con lo 
que se sienten emocionalmente involucrados si refleja sus necesidades y expectativas más genuinas. Entonces muestran disposición para acercarse, explorar, interrogar, comparar, ensayar, intercambiar. Se produce el conflicto cognitivo y la necesidad de resolverlo a través de la acción transformadora, planteado por J. Piaget, lo que solo es posible cuando en el proceso de formación de los profesionales, durante el desarrollo de las situaciones que enfrentan los estudiantes y el profesor, logran un acoplamiento a nivel emocional y a nivel cognitivo.

Disposición para aprender responsablemente. Una vez en el proceso de aprender, los estudiantes requieren mostrar y consolidar ciertas disposiciones subjetivas, características de toda situación asumida como desafío: perseverancia, tenacidad, tolerancia al fracaso, flexibilidad, control de los impulsos, etc. Es decir, el interés no basta. Para sostener con éxito la participación al interior del proceso se hace necesario desplegar, complementariamente, otras actitudes.

Disposición para desempeñarse bien en un campo. Pero el desempeño eficiente en un campo específico depende también de ciertas disposiciones afectivas coherentes con la naturaleza misma de lo que se aprende. Más allá de la implicación subjetiva en una experiencia de aprendizaje, el desempeño óptimo en un ámbito requiere una disposición especial que nos lleva a buscar nuevas oportunidades y mayores retos en ese campo en particular.

El concepto de competencia debe transmitir claramente la idea de que los procesos educativos tienen que estar dirigidos fundamentalmente a la formación de un ciudadano que reúna las condiciones que la sociedad actual está demandando, es decir, que sean individuos con capacidad plena para el análisis, la argumentación, preparados para asumir los desempeños laborales que las distintas profesiones requieren; sujetos altamente comprometidos con la historia y las tradiciones de su medio, profundamente reflexivos, capaces de comportarse esencialmente humanos, con todas las posibilidades para insertarse en el vertiginoso avance de la ciencia y la técnica y dispuestos a crecer tanto en el orden de la preparación técnica y profesional como en sus condiciones personales y espirituales.

Se puede entender la competencia profesional como una combinación de conocimientos, habilidades, comportamientos y actitudes que se pueden demostrar en un contexto profesional determinado y que se pueden transferir a diferentes condiciones a través de la actualización permanente.

Entonces, un profesional competente es:

- Aquel que se apropia de las teorías, las leyes, los conceptos, las definiciones de la rama del saber en las cuales se desenvuelve, para poder actuar responsablemente $\mathrm{y}$ con posibilidades de hacer análisis, reflexiones, inferencias, inducciones, deducciones.

- Que es hábil en su desempeño, mostrando destrezas, tanto orales y comunicativas como manuales, físicas y motoras.

- Quien se empeña por hacer las cosas bien porque está totalmente motivado con su profesión, porque lo hace conscientemente, demostrando en su desempeño profesional la convicción que tiene de la importancia social de su labor, con lo cual contribuye al desarrollo del país.

- Demuestra haberse apropiado de los aspectos más trascendentales que identifican la ética de su profesión.

- Quien es capaz de trascender sus propios aprendizajes siendo capaz de potenciar sus recursos personales y dar solución creadora a nuevos problemas profesionales. 
- Quien tiene la posibilidad de hacer transferencias de unos contenidos ya dominados a otros nuevos por medio del autoaprendizaje, del adecuado manejo de la información.

Al irse produciendo la transformación del perfil de la fuerza de trabajo, por un lado hay un acelerado incremento de la demanda de profesionales con habilidades de alto nivel técnico, en particular con las competencias necesarias para la aplicación de las tecnologías de información, y por el otro, los cambios en las habilidades requeridas para el manejo de nuevos métodos y sistemas de producción, lo que reclama de la formación del futuro egresado de la educación superior, el desarrollo de competencias profesionales.

Un plan de estudio basado en competencias evidencia una coherencia que asegurará, en una proporción considerable, que su implementación garantice los propósitos trazados siempre que dichas competencias se conviertan en el aspecto regulador del proceso de formación de los profesionales.

En consecuencia, el plan de estudio permitirá que los estudiantes del programa se identifiquen durante todo el desarrollo del mismo con las particularidades del ejercicio profesional a través de las competencias que pondrán de manifiesto y que les permitirá una integración efectiva y eficiente a su trabajo profesional una vez estén graduados.

Es indispensable construir y fortalecer un tejido social propicio a la excelencia en el desempeño de los profesionales, lo cual está asociado a demostrar las competencias laborales. La relación más estrecha entre educación y competencias se da en la educación superior.

Los cambios del currículo universitario en busca de una integralidad en el profesional se fundamentan en la necesidad de ofrecer una formación para la vida, esto es:
- Unos contenidos que reflejen de manera pertinente y relevante los cambios que ocurren en la realidad nacional e internacional, ofreciendo la posibilidad de desarrollar plenamente todas las potencialidades y capacidades para aprender a lo largo de la vida.

- Una formación que, en particular, los dote de un carácter ético centrado en el desarrollo personal en el sentido de la trascendencia, el respeto al otro, a la convivencia democrática; el respeto a la naturaleza, el amor a la verdad, la justicia y la belleza; el espíritu emprendedor y el sentimiento patriótico, de su identidad y tradiciones.

En otras palabras, para configurar actualmente un currículo, se parte de preguntas al interior de la misma institución, ideándolo según las necesidades actuales para formar y preparar estudiantes que se desempeñen en el siglo XXI.

\section{Modelo curricular para la formación por competencias en la educacion superior}

Los fundamentos didácticos de la educación superior cubana reconocen el proceso de formación de los profesionales como el objeto de la didáctica de la educación superior como ciencia, pudiéndose identificar dos ideas rectoras que permiten explicar el proceso de formación de los profesionales. Considerar que dicho proceso es un sistema de actividades conscientes, de naturaleza holística y dialéctica; y que constituye una configuración de orden superior, síntesis de expresiones dinámicas de su totalidad, que se integran en torno a los significados y sentidos que para los estudiantes tiene el proceso formativo (Fuentes, 2001). Estas ideas rectoras permiten explicar el proceso de formación de los profesionales como una totalidad y a partir de relaciones dialécticas, que expresan las regularidades del mismo.

Siendo consecuente con la concepción totalizadora de la profesión, se entiende la 
necesidad de contar con una categoría que sea la expresión didáctica de la misma y del profesional a partir de la cual el diseño de la carrera, que se realiza a nivel de macrodiseño curricular, pueda ser estructurado didácticamente a nivel del microdiseño curricular. Para poder desarrollar el proceso de formación de los profesionales en términos de contenidos en su relación con las restantes configuraciones que están presentes en este diseño. Las competencias profesionales devienen, entonces, en el eje curricular que permite trasladarse desde la profesión al proceso de formación del profesional, asumiendo el término 'eje' como el aspecto esencial para generar movimiento o el fundamento de un razonamiento o comportamiento determinado, atravesando todo el currículo y expresándose en contenidos (conocimientos, habilidades y valores profesionales) del microdiseño curricular.

Al ser las competencias profesionales una configuración didáctica integradora de la profesión y del tipo de profesional que se aspira a formar, permite precisar aquellas cualidades esenciales para el desempeño laboral de los futuros egresados, necesarias para enfrentar no solo los problemas profesionales de manera pertinente sino también para definir y realizar su proyecto de vida en el contexto social, para lo cual se requiere que el proceso de formación del profesional sea integrador del saber, el saber hacer el ser, el saber convivir y el saber emprender, en tanto los pilares necesarios para la educación definidos por Unesco.

Cualquiera que sea el modelo curricular asumido, es esencial concebir una formación profesional centrada en el desarrollo humano y que desde una concepción interdisciplinaria se garantice una sólida cultura científico-técnica, humanista y ambiental, independientemente del perfil profesional.

En las competencias profesionales se expresan los fundamentos teóricos del currículo, entendidos estos como el marco o posición que sustenta el modelo asumido, y que permite orientar la actividad del diseño curricular a través del empleo de una metodología.

Las competencias profesionales son justamente la expresión didáctica de la profesión y del profesional, en la cual se expresará la concepción curricular que permita estructurar los planes y programas de estudio y tiene la peculiaridad de ser síntesis de todos los fundamentos teóricos del currículo.

En las competencias profesionales se sintetizan las dimensiones que explican cómo se produce el movimiento del compromiso, flexibilidad y trascendencia, cualidades que deben caracterizar al profesional. Por tanto, el diseño curricular debe pretender que esas cualidades se manifiesten en el propio proceso.

Una alternativa novedosa de modelo de diseño curricular la constituye la formación por competencias, que permite la flexibilidad en el diseño de los planes de estudios y la consideración de las cualidades que deberán alcanzar los egresados desde el diseño; incorporar las competencias al proceso de concepción curricular es una manera de integrar ese concepto al de las configuraciones ya trabajadas en modelos de diseños curriculares anteriores: problema, objeto, objetivo, contenido y método (Cruz y Fuentes, 1998).

Se entiende entonces la competencia profesional como la configuración didáctica que sintetiza la riqueza de la profesión y del profesional, en tanto resultado de las relaciones dialécticas que se establecen entre el problema profesional, el objeto de la profesión y el objetivo del profesional, siendo expresión totalizadora de las cualidades que debe poseer el egresado para su desempeño profesional $\mathrm{y}$ social en un contexto histórico concreto y permitiendo generar el proceso de desarrollo del diseño curricular. 


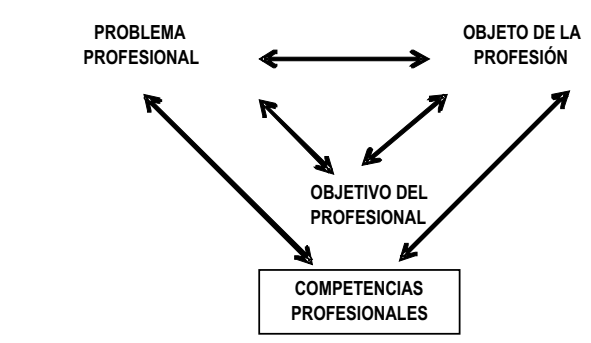

Figura 1: Concepción de las competencias profesionales como sintesis del problema, objeto y objetivo.

\section{Macrodiseño curricular}

El macrodiseño curricular es el proceso en que se definen, de conformidad con la relación que expresa el vinculo de la universidad con la sociedad, los problemas profesionales, el objeto de la profesión y el objetivo del profesional, lo que constituye el punto de partida del proceso de formación de los profesionales y para elaborar el modelo de los mismos (figura 1).

Se define el Modelo del Profesional, es decir, se determinan los aspectos más generales $\mathrm{y}$ trascendentes que deben ser incorporados al proceso de formación con la intención de crear profesionales adecuados para el desempeño social y profesional, lo que constituye el resultado del proceso curricular en el que son delimitadas las competencias profesionales.

La determinación del modelo del profesional se lleva a cabo como consecuencia de la relación entre el objetivo perseguido, el problema y el objeto de la profesión; todo lo cual permite la determinación de sus competencias.

El problema profesional es la categoría que manifiesta la necesidad de que el profesional intervenga sobre una situación dada y la transforme. El problema profesional está en la sociedad, pero cuando es delimitado en el propio proceso de formación de los profesionales se convierte en una configuración del mismo.

El objeto de la cultura de la profesión, como categoría, expresa aquella parte del fin de la ciencia, la tecnología, el arte, tradiciones o creencias, que tiene que ser incorporado al proceso de formación de los profesionales, que son expresión sintetizada del aval cultural en el cual se debe formar el profesional.

El objeto de la cultura que se tiene que traer a la formación del profesional no puede ser visto solo desde la mirada estrecha de una profesión. Por el contrario, ha de armonizar la cultura específica del objeto de la misma, con una amplia cultura general. De la contradicción entre lo estrecho de los problemas profesionales y lo amplio del objeto de la cultura se delimita el de la carrera, lo que en el microdiseño curricular se expresa en los contenidos de los programas de disciplinas y asignaturas que concretan las competencias en los conocimientos, habilidades y valores que se formarán en los estudiantes.

El objetivo del profesional expresa el resultado ideal de su proceso de estructuración, esto es, la aspiración de forjar en las instituciones de educación superior, una persona integral, que responda con calidad al encargo social cuando actúa sobre el objeto de la carrera.

Se comprende como profesional integral el que se forma: comprometido social y profesionalmente, flexible y trascendente, como esencia de los valores de un profesional y ello unido a una sólida formación de conocimientos y habilidades.

La relación dialéctica entre las configuraciones problema profesional, objeto de la profesión y objetivo del profesional constituyen una configuración de orden superior que expresa la profesión, en términos de las competencias profesionales, como la categoría la cualidad del proceso.

El Modelo Curricular para la formación por competencias parte del supuesto de que si el proceso de formación del profesional se diseña a partir de un modelo de competencias profesionales, que son expresión del compromiso, 
la trascendencia y la flexibilidad como cualidades más generales del profesional, se puede contribuir a formar un egresado comprometido social y profesionalmente, flexible en el manejo de la cultura y trascendente en su contexto, lo que se expresará en el desarrollo de valores y las actitudes que le permitan ser participativos, reflexivos, negociadores, críticos, responsables, creadores y fundamentalmente humanos ante la solución de los problemas que emanan de la profesión.

Es por eso que las dimensiones que emergen del proceso de macrodiseño, al relacionar dialécticamente el problema, el objeto y el objetivo del profesional, que se sintetizan en las competencias profesionales, son precisamente el compromiso, la trascendencia y la flexibilidad.

El compromiso laboral y social es la dimensión que adquiere la competencia profesional, expresada en su actuación cívica, activa y responsable, donde a partir de los objetivos del sujeto se enfrentan y resuelven los problemas. (Figura 2).

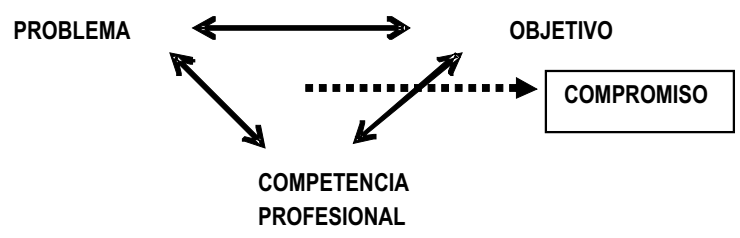

Figura 2: Dimensión de compromiso

En la concepción del profesional, expresada en la competencia laboral, se precisan las necesidades sociales que requieren de la actuación de estos, dando lugar a los problemas laborales, lo cual condiciona la determinación del tipo de profesional, que como aspiración se requiere para satisfacer esa necesidad social. A su vez, la precisión del objetivo del profesional, en tanto resultado final que aspira alcanzar, se revela en la competencia, el compromiso social que asume respecto al problema. De lo que se trata es de forjar un profesional que resuelva la necesidad social que le da origen a la profesión, por lo que el eje curricular revela la cualidad de compromiso social que desde la profesión asume el capacitado, de manera que esta cualidad se denomina compromiso profesionalsocial, haciendo connotar esa particularidad.

La competencia profesional adquiere la dimensión de compromiso social cuando la aspiración formativa expresada en el objetivo del egresado es contentiva de los valores que caracterizan su actuación, en función de las necesidades sociales representadas en el proyecto en que se inserta, y que se configura en los problemas laborales, lo cual posibilita que el proceso formativo propenda a que la carrera adquiera significado y sentido para el egresado y actúe de manera comprometida con la sociedad. No se puede perder de vista que es la sociedad quien le demanda a la universidad el tipo de profesional que necesita, sintetizado didácticamente en el problema y en el objetivo $\mathrm{y}$ es el currículo quien tiene que asumir ese imperativo.

Al tener la competencia profesional una dimensión de compromiso social, es posible entonces diseñar un currículo que permita formar un graduado con la cualidad de compromiso profesional-social, propiciando que pueda insertarse conscientemente, desde su área de trabajo, en el proyecto social. De ser así, la competencia laboral propende a que el proceso de formación de los egresados pueda cumplir una función educativa.

Se define la cualidad de compromiso profesional-social como "las peculiaridades de actuación profesional y cívica activa y responsable en el proyecto social en que se inserta el graduado, a partir de los significados y sentidos que tiene para él la carrrera y su lugar en el proyecto social. Significa entonces que desde la concepción del profesional se debe proyectar la preparación de los egresados para esa participación, desarrollando las competencias que necesita. 
Cuando José Martí plantea que educar «es preparar al hombre para la vida» encierra la cualidad de compromiso social del profesional que debe asumir ante la vida"(Valera, 2003).

En la relación dialéctica que se establece entre las configuraciones problema profesional, objeto de la carrera y la competencia laboral, se adquiere la dimensión de trascendencia curricular, revelando la cualidad trascendente del profesional (Figura 3).

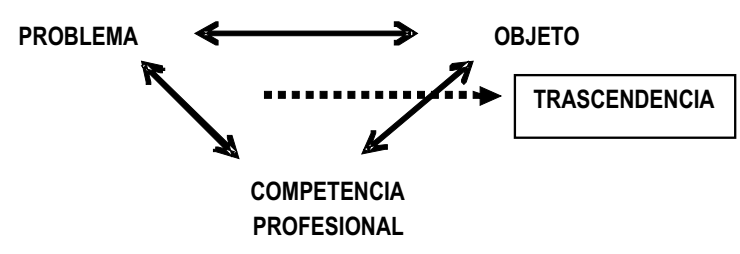

Figura 3: Dimensión de trascendencia

La trascendencia es la dimensión que adquiere la competencia profesional expresada en la creatividad e innovación del graduado, donde a partir del objeto de la carrera se enfrentan y resuelven los problemas.

Desde la concepción del profesional, expresada en la competencia, se debe tener en cuenta la parte de la cultura que se necesita para poder resolver los problemas laborales, en tal sentido, el objeto de la carrera está condicionado por los problemas profesionales como expresión de la necesidad social. A su vez, los problemas profesionales se condicionan al propio desarrollo de la cultura y su delimitación en el objeto de la profesión.

En la relación dialéctica entre el problema y el objeto de la profesión, la competencia profesional adquiere la dimensión de trascendencia cuando la parte de la cultura que se delimita para la solución del problema, como expresión de la necesidad social, garantiza una profunda formación básica de sus competencias, con lo cual se contribuye a desarrollar las potencialidades transformadoras del egresado, preparándolo suficientemente para encontrar soluciones a las necesidades sociales actuales, a la vez que se garantice su desarrollo futuro, posibilitando delimitar y enfrentar nuevos problemas en correspondencia con las necesidades siempre cambiantes de la sociedad.

Significa entonces que la competencia profesional debe revelar la dimensión de trascendencia y en correspondencia con esto se debe diseñar un currículo que propenda a la formación de un graduado trascendente a su tiempo, propiciando el desarrollo de sus capacidades creativas y transformadoras. De ser así, la competencia laboral posibilita que el proceso de formación cumpla con su función desarrolladora.

Se entiende la cualidad trascendente del profesional como la peculiaridad creativa, innovadora y transformadora alcanzada, que propende al desarrollo humano y que permite una proyección que rebase los marcos actuales de desempeño.

Esta cualidad trascendente del profesional queda expresada genialmente por José Martí cuando al definir la educación del hombre plantea que «es ponerlo a nivel de su tiempo para que flote sobre él y no dejarlo debajo de su tiempo, con lo que no podría salir a flote»" 3 .

Al establecerse la relación dialéctica entre el objetivo del profesional y el objeto de la profesión, la competencia de la carrera adquiere la dimensión de flexibilidad curricular, revelando la cualidad flexible del graduado. (Figura 4)

OBJETIVO

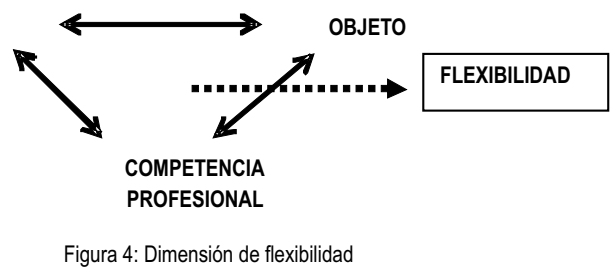

La flexibilidad es la dimensión que adquiere la competencia profesional expresada 
en la búsqueda de alternativas y protagonismo, donde a partir del objeto de la carrera, se enfrenta el objetivo del graduado.

En el proceso de concepción de la profesión, expresado en la competencia laboral, se delimita aquella parte de la cultura que se necesita para resolver el problema profesional, configurado en el objeto de la carrera, de acuerdo con la aspiración del tipo de graduado que se requiere para resolver dicho problema, configurado en el objetivo. En este caso, el objeto de la profesión está condicionado por el objetivo del profesional. A su vez, para precisar el objetivo alcanzado en el resultado esperado, se busca resolver la necesidad social, tomando en cuenta el nivel de desarrollo logrado.

La competencia profesional adquiere la dimensión de flexibilidad cuando el objetivo del profesional, que expresa la aspiración del graduado, que se requiere para resolver las necesidades de la sociedad, propicia la incorporación al currículo de nuevos elementos de la cultura que amplían y enriquecen el objeto de la profesión, con lo cual se logra mayor sistematización y actualización de los contenidos. Esto implica que a partir de una formación básica, que incluye los aspectos esenciales e indispensables para la actuación del profesional, se incorporen aspectos de la cultura científica, técnica, humanística y ambiental que, unidas al objeto de la profesión, permitan profundizar, actualizar y ampliar su preparación.

Lo anteriormente planteado lleva a considerar que la competencia profesional debe contener la dimensión de flexibilidad, y consecuentemente el currículo que se diseñe deberá ser susceptible de modificaciones y mejoras constantes de acuerdo con el desarrollo de la cultura necesaria para la profesión, con lo cual se contribuye a las capacidades cognitivas del profesional en formación. El deslinde de lo que se puede considerar básico para la formación de los graduados es importante y debe corresponder con las necesidades siempre cambiantes de la sociedad; en todos los casos, independientemente del tipo de diseño curricular que se asuma, debe garantizarse la correspondencia del objeto de la profesión con el objetivo del profesional.

Se puede decir que la flexibilidad de la competencia profesional posibilita que el estudiante universitario pueda asumir una posición protagónica en su propia formación, estando en capacidad de decidir algunos de los contenidos de su currículo a partir de conocer el objetivo profesional, en correspondencia con el objeto de la profesión. La flexibilidad se expresa además en el hecho de que el profesional, una vez egresado del centro de educación superior, esté en condiciones de adaptar y renovar los conocimientos que son necesarios para mantener su profesionalidad.

El hecho de que la competencia profesional permita diseñar un currículo flexible favorece que el proceso de formación de los profesionales pueda cumplir la función instructiva.

"La cualidad flexible del profesional se entiende como la peculiaridad de análisis histórico concreto y búsqueda de alternativas de solución a problemas profesionales que requieren de su actuación, sin que se pierda la finalidad. El estudiante puede asumir una posición activa y protagónica, cuando el currículo está diseñado para que el estudiante universitario, a partir de sus expectativas y necesidades, lo construya y personalice.

Cuando José Martí plantea que «educar es hacer a cada hombre resumen del mundo viviente, hasta el día en que vive», está expresando la necesaria flexibilidad que debe tener el proceso formativo, que permita formar en el hombre la cualidad flexible que se requiere para una educación a lo largo de la vida, permitiendo la construcción continua de conocimientos y habilidades necesarios para desempeñar su función social profesional y cívica"4. 
Se pretende que desde la propia concepción de la profesión y del profesional, se lleve al currículo aquellas cualidades que se necesitan formar y que podrán ser constituídas en la dinámica del proceso de formación de los graduados.

El proceso de formación de los profesionales debe ser diseñado con las tres dimensiones integradas dialécticamente, lo cual permitirá desarrollar en un proceso único y totalizador las funciones de instrucción, educación y desarrollo, a través de las cuales podamos formar un egresado comprometido, flexible y trascendente.

Para que el proceso de formación de los profesionales se desarrolle es necesario que el diseño del currículo, de acuerdo con el nivel de sistematicidad del contenido, transite en orden descendente desde el nivel de carrera, donde a través del macrodiseño se obtiene el Modelo del Profesional como documento que formaliza el currículo a este nivel, hasta los niveles de disciplinas, asignaturas y temas donde por medio del microdiseño se obtienen los programas correspondientes.

Las competencias son configuraciones que didácticas sintetizan lo cognitivo, lo axiológico y lo actitudinal, lo que conlleva las destrezas y capacidades necesarias. En la formación de un profesional se atiende a tres tipos de competencias a saber: competencias profesionales, competencias básicas y competencias generales.

Las competencias profesionales son aque1las expresiones didácticas de las cualidades del sujeto, en que se sintetiza el ser, saber y el hacer del profesional, así como el desarrollo de sus capacidades y aptitudes, al desempeñarse en los procesos profesionales.

Las competencias básicas son aquellas expresiones didácticas de las cualidades del sujeto, en que se sintetiza el ser, saber y el ha- cer, así como el desarrollo de las capacidades y aptitudes del profesional universitario y que sustentan el desarrollo de las competencias profesionales, siendo estas competencias comunes a diversos profesionales.

Las competencias generales son aquellas expresiones didácticas en que se sintetiza el ser, saber y el hacer así como el desarrollo de las capacidades y aptitudes del profesional al desempeñarse como tal en el ámbito social, cultural y laboral, siendo de carácter humanístico e investigativo.

En tal sentido, el modelo de diseño curricular que se presenta, privilegia el desarrollo de las competencias profesionales como ejes curriculares que atraviesan el proceso de diseño, expresando el desempeño que se aspira del egresado como totalidad.

En la Educación Superior, los esfuerzos encaminados a elevar la pertinencia, el impacto y la optimización de todo el proceso están estrechamente ligados al fortalecimiento de la eficiencia, la eficacia, la efectividad y la expectatibilidad del proceso de formación de los profesionales, lo que significa mejorar su respuesta a las necesidades de la sociedad, su relación con el sector productivo, asistencial y de servicios, así como su contribución a un desarrollo humano sustentable, y ello es posible cuando las cualidades que distinguen al profesional son su compromiso social y laboral, su flexibilidad y su trascendencia.

De igual modo, se determina el perfil ocupacional con el que se identifican los lugares en que se empleará o que podrá desempeñarse el egresado, así como los modos de actuar más comunes y frecuentes interpretados estos como la generalización de los métodos que en la actualidad desarrollan los profesionales con mayor frecuencia. Lo anterior no significa que se esté concibiendo un profesional que exclusivamente pueda desempeñarse en los lugares donde actualmente trabaja y empleando 
los métodos que hoy se aplican, pero ello constituye un punto de referencia necesario para poder formar ese profesional flexible y trascendente que es requerido.

Para ello es necesario establecer un programa de académico que fomente las competencias de los estudiantes, no solo en los contenidos específicos de la profesión, sino en la formación general que comprenda los aspectos sociales, humanísticos e investigativos en las ciencias básicas. Así como en aquellos conocimientos que conforman su acervo cultural; favoreciendo los contenidos tratados de modo interdisciplinario y transdisciplinario.

El macrodiseño curricular se culmina cuando se ha determinado el Modelo del Profesional, lo que implica determinar las competencias profesionales, básicas y generales; precisar los créditos por componentes de formación general, básico, básico profesional y profesional específico, los que se han de llevar a créditos por ciclos del plan de estudios como son los generales, profesionales y de profundización, todo lo cual permite valorar y balancear la formación del profesional, lo que permitirá elaborar el gráfico semestral de asignaturas. Los procedimientos del macrodiseño y los instrumentos aparecen en la metodología del mismo.

Para poder concluir el macrodiseño curricular se requiere de haber elaborado el microdiseño de componentes y núcleos de contenido, en el que se determinan los tiempos requeridos para el desarrollo y apropiación de los contenidos y, en consecuencia, los créditos.

Las consideraciones anteriormente planteadas son necesarias para comprender que esta nueva manera de asumir las competencias profesionales como configuraciones didácticas de la profesión y del profesional, expresadas en cualidades genéricas de compromiso, flexibilidad y trascendencia, tiene su concreción particular en el diseño de la profesión y del profesional en particular de que se trate, lo que le aporta espe- cificidad y ellas mismas son expresiones de las anteriores cualidades definidas. La formulación de las competencias profesionales que hace en el macrodiseño debe realizarse en términos de las cualidades de compromiso, flexibilidad y trascendencia profesional a partir de la riqueza de la profesión, esto es, se precisa quién es ese profesional que se quiere formar, qué lo cualifica, considerando la interacción del problema profesional, el objeto de la profesión y los objetivos del profesional. Esta concepción trasciende la posición reduccionista de asociar la competencia solo como un aspecto que los que configuran la profesión y el profesional, a saber, el objetivo, o los que la identifican con habilidades y su derivación en formación de capacidades.

Siendo así, se preserva el carácter holístico totalizador de las competencias profesionales expresadas en el modelo del graduado y su traslado al microdiseño se expresa en todas las configuraciones que interactúan en ese eslabón del diseño. Las competencias profesionales estarán contenidas en los programas de disciplinas y asignaturas en términos de problema, objeto, objetivo, contenido y método, orientados al proceso formativo para alcanzar las cualidades definidas, las que estarán presentes en cada una de las cualidades que emergen de las dimensiones del microdiseño y que a continuación se explican.

\section{Microdiseño curricular}

El modelo curricular se complementa con el microdiseño curricular de los componentes, donde se determinan los núcleos de contenido que se integran en los elementos de formación del programa académico. Los núcleos de contenido a su vez están conformados por núcleos de conocimiento, los invariantes de habilidad y los valores profesionales que se propician formar desde estos núcleos.

Determinar la estructura de los núcleos de conocimiento, los invariantes de habilidad e incluso los valores profesionales permite 
disponer de los elementos de juicio necesarios para determinar los tiempos y créditos del programa académico.

En la determinación de los contenidos se realiza a partir del objeto y se explica desde las relaciones entre las configuraciones del proceso de formación del profesional, las que precisan las dimensiones del mismo.

La primera de estas dimensiones es la que se da respecto al problema, y va a determinar qué aspectos del objeto de la cultura se traen al contenido en aras de resolver el problema planteado, esta es la dimensión gnoseológica. (Figura 5).

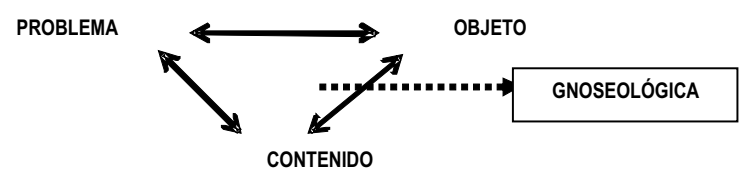

Figura 5: Dimensión gnoseológica del contenido

De la relación dialéctica entre las configuraciones objeto y problema, el contenido adquiere la dimensión gnoseológica, revelando la cualidad cognoscitiva del estudiante.

Lo gnoseológico es la dimensión que adquiere el contenido expresada en su cognoscibilidad, cuando delimita el objeto de la cultura para enfrentar y resolver problemas.

En el contenido se delimita la parte de la cultura que se necesita en el proceso docente educativo para que el estudiante pueda enfrentar los problemas de la profesión, expresados en problemas docentes en estos niveles de precisión de la carrera y en tal sentido, el contenido es gnoseológico, quiere decir que el contenido incluye los conocimientos, habilidades y valores que necesitan apropiarse los estudiantes para poder resolver los problemas docentes.

Por cualidad de cognoscibilidad del contenido se entiende la peculiaridad de ser apropiado por el estudiante.
Ahora bien, el contenido no incluye solo el aspecto gnoseológico, dado que la solución de los problemas se enfrenta desde las competencias como expresión de la generalización del contenido, sino que está presente la dimensión profesional. (Figura 6).

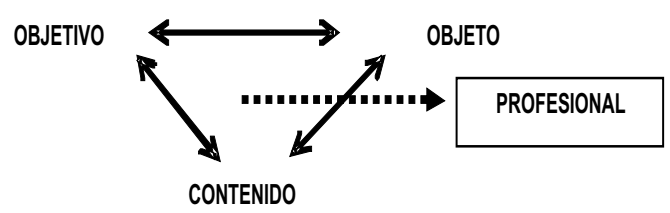

Figura 6: Dimensión profesional del contenido

En la relación dialéctica que se establece entre el objeto de la cultura y el objetivo, que expresa el profesional, el contenido adquiere la dimensión laboral, revelando la cualidad de idónea del estudiante.

Lo profesional es la dimensión que adquiere el contenido, expresada en su utilidad laboral, cuando se delimita el objeto para alcanzar el objetivo.

En el contenido se delimita aquella parte de la cultura que se requiere en el proceso docente educativo para dar respuesta a la formación profesional que se aspira, expresado en el objetivo. En tal sentido, el contenido, como expresión de la concepción de la profesión en los niveles de área o disciplina y asignatura o módulo, es profesional. Significa que el contenido incluirá los conocimientos, habilidades y valores profesionales que tienen quedesarrollarseen losestudiantesuniversitarios para que estos adquieran profesionalidad. Por cualidad de profesionalidad del contenido se entiende la peculiaridad de utilidad profesional para el estudiante, expresado en términos de los conocimientos, habilidades y valores que se necesitan para alcanzar el objetivo.

La profesionalidad como cualidad que se revela en la dimensión profesional del contenido asume de manera integradora las cualidades expresadas en la concepción de la profesión. 
En la determinación del contenido, además de las dimensiones gnoseológica y profesional, hay que considerar la de comprensión de ese contenido, el que debe ser configurado de manera tal que se puede comprender y posibilitar, por tanto, su apropiación a través del proceso. La dimensión metodológica, que expresa la comprensión y que está dada por la relación entre el objeto y el método, que se sintetiza en el contenido. (Figura 7).

De la relación dialéctica entre las configuraciones objeto y método, el contenido adquiere la dimensión metodológica, revelando la cualidad de comprensibilidad del estudiante.

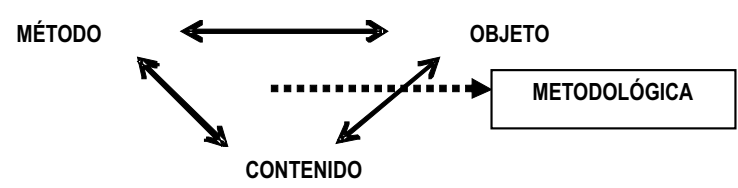

Figura 7: Dimensión metodológica del contenido

Lo metodológico es la dimensión que adquiere el contenido expresado en su secuencia y lógica cuando delimita el objeto de cultura para ser comprendido.

En el contenido se delimita la parte de la cultura que se necesita en el proceso docente educativo y que el estudiante debe comprender, para lo cual deberá recurrir a determinados métodos, es decir, que el contenido debe expresarse en términos de lo que el estudiante pueda comprender, estructurándose la secuencia o vía para lograrlo.

El método es la expresión del proceso que se configura en la relación proceso-sujeto, siendo la vía que se adopta en la ejecución del mismo, por los sujetos que lo realizan para que a través del contenido, alcancen el objetivo. Con el método se sintetiza la acción intelectual, motivacional y afectiva de los sujetos.

Por cualidad de comprensibilidad del contenido se entiende la peculiaridad de su instrumentación lógica para el estudiante.
Resumiendo, en estas tríadas se expresa el proceso de tránsito de objeto a contenido que se desarrolla en el microdiseño curricular que, como se ha visto, requiere de una dimensión gnoseológica, que está en la cultura, a la que se incorpora una dimensión profesional, que está en las competencias profesionales, y una dimensión metodológica o de comprensión del contenido. Las relaciones entre estas configuraciones revelan las regularidades con que se elabora el contenido de los programas de las disciplinas y asignaturas o módulos. Un contenido que es expresión de las competencias profesionales en los niveles de sistematicidad de la profesión que precisan la carrera, que en su papel mediador en las relaciones que se establecen entre determinadas configuraciones revela las dimensiones profesional, metodológica y gnoseológica del proceso.

El modelo curricular se articula con la aplicación de métodos pedagógicos y didácticos que propicien una efectiva inserción de los egresados en su ejercicio profesional, teniendo en cuenta la rapidez con que se producen los avances en el campo de la profesión, y en particular el incremento incesante de las tecnologías de la información y la comunicación.

\section{Conclusiones}

Se comprende como profesional integral el que se forma: comprometido social y profesionalmente, flexible y trascendente, como esencia de los valores de un profesional y ello unido a una sólida formación de conocimientos y habilidades.

En última instancia, el término 'competencias' intenta recoger la dialéctica que se produce en la relación hombre-mundo y que se expresa simultáneamente como exigencia de un desempeño profesional y las cualidades personales para realizar esa actividad.

El proceso de formación del profesional basado en competencias, que se desarrolla en 
la universidad, parte del supuesto de que si el proceso de formación del profesional se diseña a partir de un modelo de competencias profesionales, que son expresión del compromiso, la trascendencia y la flexibilidad como cualidades más generales del profesional, se puede contribuir a formar un egresado comprometido social y profesionalmente flexible en el manejo de la cultura y trascendente en su contexto, lo que se expresará en el desarrollo de valores y las actitudes que le permitan ser participativos, reflexivos, negociadores, críticos, responsables, creadores y fundamentalmente humanos, ante la solución de los problemas que emanan de la profesión.

\section{Notas}

${ }^{1}$ J. Gimeno Sacristán. 'Políticas y prácticas culturales en las escuelas: los abismos de la etapa postmoderna'. Trabajo solicitado por Heuresis.

${ }^{2}$ Ibídem, p. 62

${ }^{3}$ René Valera, ob.cit. P. 55

${ }^{4}$ René Valera, ob.cit. P. 57

\section{Bibliografía}

Colectivo de autores (2000). El currículo hoy: realidad y perspectiva en Cuba. Centro de Estudio de Educación, Ispejv. La Habana, Cuba.

Corral R. (1990). Aplicación de un método teórico a la elaboración del perfil profesional en la educación superior. $R e$ vista cubana de Educación Superior. X (2). La Habana, Cuba.

Corral, R. (2004). El currículo basado en competencias. Ponencia, Universidad de La Habana.
Cruz S. y Fuentes H. (1998). El Modelo de Actuación Profesional: una propuesta viable para el diseño curricular de la educación superior. CEES, Universidad de Oriente, Santiago de Cuba.

Díaz B., A. (1992). Ensayos sobre la Problemática Curricular. Editorial Trillas, México.

Díaz B., F. (1996). Metodología del diseño curricular para la Educación Superior. Editorial Trilla, México.

Fuentes, H. (1995). Fundamentos didácticos para un proceso de enseñanza - aprendizaje participativo. CEES, Universidad de Oriente, Santiago de Cuba.

Fuentes, H. (1998): Modelo Holístico Configuracional de la Didáctica de la Educación Superior. CEES. Universidad de Oriente. Santiago de Cuba.

Fuentes, H. (2001). Bases epistemológicas y metodológicas de la teoría holístico configuracional de los procesos sociales. Soporte magnético, CEES, Universidad de Oriente, Santiago de Cuba.

Fuentes, H.; Cruz, S. y Valera, R (2005). Una concepción curricular basada en la formación de competencias. Monografía, CEES, Universidad de Oriente.

Horruitiner, P. (2000). El Modelo Curricular en la Educación Superior Cubana. Ponencia Central en 'Universidad 2000'. Editorial Félix Varela, La Habana.

Valera, R. (2003). Metodología para la evaluación de planes y programas de estudios de carreras universitarias. Monografía, Tesis de Doctorado. 
